\title{
Commentary: Outcomes and transferability of ex vivo lung perfusion
}

\author{
M. Shea Harrison, MD, C. Corbin Frye, MD, and Varun Puri, MD
}

\footnotetext{
From the Division of Cardiothoracic Surgery, Department of Surgery, Washington University School of Medicine, St Louis, Mo.

Disclosures: Authors have nothing to disclose with regard to commercial support.

Received for publication July 31, 2019; accepted for publication July 31, 2019; available ahead of print Oct 13, 2019.

Address for reprints: M. Shea Harrison, MD, Division of Cardiothoracic Surgery, Department of Surgery, Washington University School of Medicine, Campus Box 8234, 660 S Euclid Ave, CSRB Room 3316, St Louis, MO 63110 (E-mail: msharrison@wustl.edu).

J Thorac Cardiovasc Surg 2020;159:356-7

$0022-5223 / \$ 36.00$

Copyright (C) 2019 by The American Association for Thoracic Surgery

https://doi.org/10.1016/j.jtcvs.2019.07.125
}

The article in this issue of the Journal by Cypel and colleagues ${ }^{1}$ of Toronto presents a large cohort single-center experience with clinical ex vivo lung perfusion (EVLP). Previous studies have shown promising outcomes for EVLP to evaluate and treat donor lungs, but there has been limited worldwide experience with this relatively new technology. ${ }^{2-5}$ Most studies have had small sample sizes and have failed to answer the question of when EVLP is clinically indicated. Cypel and colleagues ${ }^{1}$ evaluated 262 lung transplants performed after EVLP and focused on the impact of indication for EVLP on lung utilization rates and transplant outcomes. They found no association between EVLP indication and short- or long-term outcomes; in addition, the introduction of EVLP was associated with a significant increase in lung transplant activity at their program. This holds promise for increased utilization of lungs, especially for donation after cardiac death lungs and lungs of questionable quality.

Two factors may be underappreciated in the interpretation of the study of Cypel and colleagues ${ }^{1}$ : the subjectivity of lung assessment and the effects of regional and institutional resources and practices in lung utilization rates. Although Cypel and colleagues ${ }^{1}$ clearly defined their indications for EVLP, wide variability exists in the assessment of donor lungs. This is reflected in the substantial variations in lung utilization rates across various regions in the United States. Institutional practices also impact lung utilization. As an example, a lung protective management protocol at a specialized donor care facility can increase the lung utilization rate to nearly double the national average. $^{6}$

Given the shortage of suitable donor lungs, EVLP has potential to increase lung utilization both through treatment and through more confident assessment of marginal lungs. The data presented by Cypel and colleagues ${ }^{1}$ suggest that EVLP helped Toronto to nearly double their transplantation

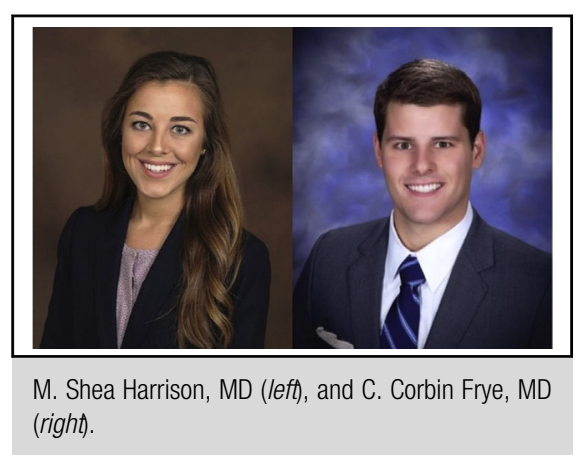

Central Message

EVLP is a powerful tool that may improve lung utilization rates and increase transplant activity; however, the transferability of these effects to transplant centers-at-large remains unclear.

See Article page 346

activity from 2008 to 2017 . The lung utilization rate, however, remained relatively stable during this time at approximately $30 \%$ to $40 \%$. Notably, this lung utilization rate is higher than that reported in the United States, which is likely related to both the application of EVLP and the effect of an experienced, high-volume center with excellent outcomes. Cypel and colleagues ${ }^{1}$ note that the increase in lung transplant offers (from 246 in 2008 to 456 in 2017) were primarily marginal organs obtained through donation after cardiac death and from elderly donors. EVLP was likely an important factor in preventing their utilization rate from declining despite lower quality offers. In addition, this article gives rise to a potential new indication for EVLP-logistic complexity - that has potential to further increase lung utilization.

The Toronto Lung Transplant Program has developed an in-house EVLP protocol that provides efficient and cost-effective EVLP. The absence of this infrastructure at other institutions could lead to a more expensive and time-consuming experience with EVLP. Although the cost of EVLP in the US and Canada has not been formally evaluated, the Donor Ex Vivo Lung Perfusion in UK lung transplantation study found that the average cost was equivalent to approximately $\$ 53,000$ when using similar methods in the United Kingdom. ${ }^{7}$ In addition, Toronto's extensive experience with EVLP likely affects their outcomes favorably, clouding the discussion of transferability 
to other institutions with less experience. Broader utilization could pose challenges of cost and time expenditure. Ultimately, further studies are needed to determine how EVLP will influence lung transplant centers at large.

\section{References}

1. Cypel M, Yeung JC, Donahoe L, Chen M, Zamel R, Keshavjee S. Normothermic ex vivo lung perfusion: does the indication impact organ utilization and patient outcomes after transplantation? J Thorac Cardiovasc Surg. 2020;159:346-55.e1.

2. D'Cunha HC, Rojas M. Ex vivo lung perfusion: past, present, and future. ASAIO J. 2018;64:135-9.
3. Tane S, Noda K, Shigemura N. Ex vivo lung perfusion: a key tool for translationa science in the lungs. Chest. 2017;151:1220-8.

4. Aigner C, Slama A, Hötzenecker K, Scheed A, Urbanek B, Schmid W, et al. Clinical ex vivo lung perfusion-pushing the limits. Am J Transplant. 2012;12: 1839-47.

5. Cypel M, Yeung JC, Liu M, Anraku M, Chen F, Karolak W, et al. Normothermic ex vivo lung perfusion in clinical lung transplantation. N Engl J Med. 2011;364: 1431-40.

6. Chang SH, Kreisel D, Marklin GF, Cook L, Hachem R, Kozower BD, et al. Lung focused resuscitation at a specialized donor care facility improves lung procurement rates. Ann Thorac Surg. 2018;105:1531-6.

7. Fisher A, Andreasson A, Chrysos A, Lally J, Mamasoula C, Exley C, et al. An observational study of donor ex vivo lung perfusion in UK lung transplantation: DEVELOP-UK. Health Technol Assess. 2016;20:1-276. 